\title{
Zum 300. Geburtstag von Leonhard Euler
}

Das letzte Heft der Elemente der Mathematik im Jahr 2007 ist dem 300. Geburtstag Leonhard Eulers gewidmet. Für dieses Heft ist es uns gelungen, fünf interessante Artikel zusammenzutragen, die auf Vorträgen beruhen, die im Verlauf dieses Jahres bei Veranstaltungen zum Euler-Jahr gehalten wurden. Das Euler-Heft beginnt mit N. Schappachers Beitrag, der uns bekannte, aber auch weniger bekannte Facetten von Eulers Werk näher bringt. Hier ist die Grundlage ein Hauptvortrag, den N. Schappacher im März dieses Jahres an der gemeinsamen Jahrestagung der Deutschen Mathematiker-Vereinigung und der Gesellschaft für Didaktik der Mathematik in Berlin gehalten hat. Der zweite Beitrag von $\mathrm{H}$. Koch beschäftigt sich mit dem Briefwechsel zwischen Euler und Goldbach. Dieser Artikel basiert auf einem Vortrag, den H. Koch anlässlich des Besuchs einer Delegation der Mathematical Association of America zum Euler-Jahr im Juli in Berlin gehalten hat. Im dritten Artikel führt uns Y.-F.S. Pétermann in Eulers Untersuchungen zur Divergenz der Reihe der Reziproken der Primzahlen ein. Der vierte Beitrag von W. Gautschi bringt uns Eulers Fähigkeiten als Numeriker im Zusammenhang mit dem Basler Problem näher, d.h. der Bestimmung des Werts der Reihe der Reziproken der Quadratzahlen. Dieser Beitrag basiert auf dem Vortrag, den W. Gautschi beim 187. Jahreskongress der Akademie der Naturwissenschaften der Schweiz im September in Basel gehalten hat. Der letzte Beitrag des Euler-Hefts von G. Ziegler und Ch. Blatter beschäftigt sich mit Eulers bekannter Polyederformel. Der Beitrag fusst auf G. Zieglers Vortrag am Internationalen Euler Symposium Ende Mai dieses Jahres in Basel.

An dieser Stelle möchte ich allen Autoren sehr herzlich dafür danken, dass sie sich bereit erklärt haben, ihren Beitrag den Elementen der Mathematik für dieses Euler-Heft zur Verfügung zu stellen. Dem Präsidenten der Schweizerischen Mathematischen Gesellschaft, N. Hungerbühler, möchte ich für die Unterstützung bei der Erstellung dieses Hefts danken. Weiter geht mein Dank an G. Szpiro für seine Zustimmung, seine in der Neuen Zürcher Zeitung erschienenen Buchbesprechungen zu neuer Literatur über Leonhard Euler in diesem Heft erneut abdrucken zu dürfen. Schliesslich geht mein Dank an das EMS Publishing House, das sich bereit erklärt hat, für dieses Heft einen zusätzlichen Seitenumfang ohne Mehrkosten zu gewähren. 